\title{
Intoxicação experimental por Dodonea viscosa (Sapindaceae) em bovinos ${ }^{1}$
}

\author{
Cristhiane Stecanella de Oliveira Cattani ${ }^{2}$, Edson Moleta Colodel2 ${ }^{2}$ Sandra Davi \\ Traverso ${ }^{2}$, André Mendes Ribeiro Correa ${ }^{2}$ e David Driemeier ${ }^{2}$
}

\begin{abstract}
Cattani C.S.O., Colodel E.M., Traverso S.D., Correa A.M.R., \& Driemeier D. 2004. [Experimental poisoning by Dodonea viscosa (Sapindaceae) in cattle.] Intoxicação experimental por Dodonea viscosa (Sapindaceae) em bovinos. Pesquisa Veterinária Brasileira 24(1):31-34. Depto Patologia Clínica Veterinária, Faculdade de Veterinária, Universidade Federal do Rio Grande do Sul, Av. Bento Gonçalves 9090, Bairro Agronomia, Cx. Postal 15094, Porto Alegre, RS 91540000, Brazil. E-mail: moleta@ terra.com.br

The leaves of Dodonea viscosa were force fed to five bovines. Four received the leaves in fresh green stage and the fifth dried ones. Clinical signs were observed in four of the bovines that died; the fifth did not show signs of poisoning. The fresh green plant was proved to be toxic from a dose of $25 \mathrm{~g} / \mathrm{kg}$ on. Dried leaves fed at the dose of $30 \mathrm{~g} / \mathrm{kg}$ were also toxic. All the animals that died showed clinical signs from 13h30min to $45 \mathrm{~h}$ after the ingestion of the plant and and death followed within about 48h. The clinical course lasted for about $8 \mathrm{~h} 30 \mathrm{~min}$ until death. The main symptoms where apathy, anorexia, slight tenesmus, muscle trembling, difficulties to keep consciousness, pressing the head against obstacles, lateral recumbency, paddling movements, coma and death. The most significant macroscopic alterations were observed in the liver, with accentuation of the lobular pattern, dark-red areas interspersed with yellowish areas. Petechiae were found in serosal membranes of the abdominal and thoracic organs as well as the intestines. The main microscopic change was hepatic centrolobular coagulative necrosis, associated with congestion and hemorrhages.
\end{abstract}

INDEX TERMS: Dodonea viscosa, diseases of cattle, plant poisoning, hepatocellular necrosis.

RESUM 0.- As folhas de Dodonea viscosa foram administradas por via oral a cinco bovinos, dos quais quatro receberam folhas verdes frescas e o quinto, folhas secas. Quatro animais apresentaram sinais clínicos e morreram, enquanto 0 quinto bovino não adoeceu. A planta verde fresca mostrouse tóxica a partir de $25 \mathrm{~g} / \mathrm{kg}$. Todos os animais que morreram, manifestaram sinais clínicos entre 13h30min e 45h após a ingestão das folhas. A evolução clínica foi de aproximadamente 8h30min. Os animais manifestaram apatia, anorexia, leve tenesmo, tremores musculares, dificuldade para permanecer em estação pressionando a cabeça contra obstáculos, decúbito esternal, movimentos de pedalar, coma e morte. A alteração macroscópica mais significativa foi observa-

\footnotetext{
${ }^{1}$ Recebido em 20 de outubro de 2003.

Aceito para publicação em 28 de novembro de 2003.

2Departamento de Patologia Clínica Veterinária, Faculdade de Veterinária, Universidade Federal do Rio Grande do Sul (UFRGS), Cx. Postal 15094, Porto Alegre, RS 91540-000. E-mail: moleta@ terra.com.br
}

da no fígado, com acentuação do padrão lobular, áreas vermelho-escuras intercaladas com áreas vermelho-claras, dando um aspecto de fígado noz-moscada. Hemorragias petequiais foram encontradas em serosas de órgãos da cavidade abdominal e torácica bem como no intestino. A principal alteração microscópica observada foi necrose hepatocelular coagulativa centrolobular ou massiva, associada à congestão e hemorragias.

TERMOS DE INDEXAÇÃO: Dodonea viscosa, necrose hepatocelular, doenças de bovinos, plantas tóxicas.

\section{INTRODUÇÄO}

Dodonea viscosa (L.) Jacq. (Fig. 1 e 2), vulgarmente chamada de vassoura-vermelha, vassoura-do-campo ou erva-de-veado, é uma planta nativa sucessora, sendo uma das primeiras espécies a povoar áreas desmatadas. No Brasil, Dodonea viscosa ocorre ao longo de todo o litoral da Região Sul (Reitz 1980). É usada como planta ornamental e medicinal. Isolou-se de D. viscosa saponinas e glicosídeos cianogênicos (Simões et al. 


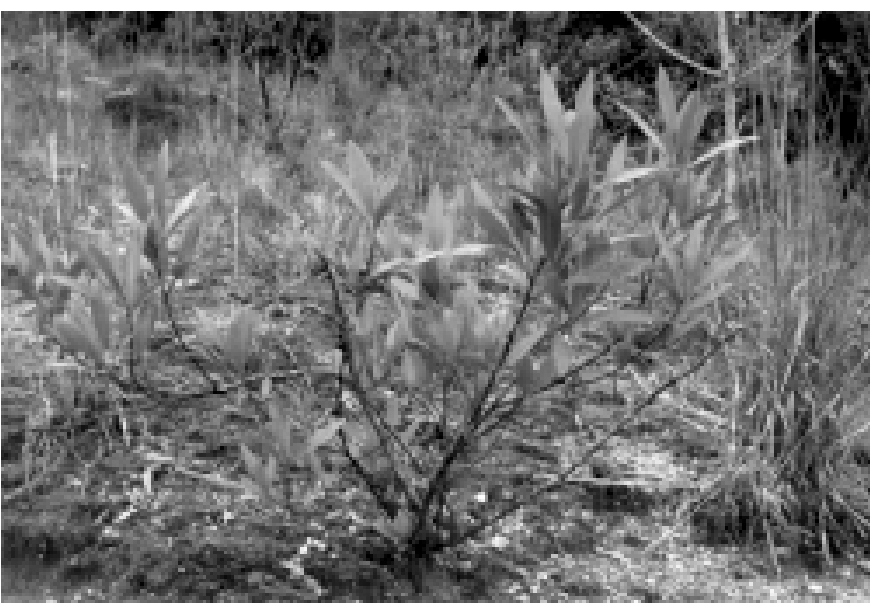

Fig. 1. Dodonea viscosa (vassoura-vermelha), em setembro, Porto Alegre, RS.

1989, Mors et al. 2000, Pesman \& Ervin 2002) que não foram associados com intoxicação em animais domésticos.

A intoxicação natural por D. viscosa foi associada com a mortalidade em um rebanho bovino no Estado do Rio Grande do Sul (Colodel et al. 2003).

0 desconhecimento sobre a intoxicação por $D$. viscosa em animais domésticos tornou oportuna a realização deste estudo experimental para caracterização das doses tóxicas e do quadro clínico e patológico em bovinos intoxicados com folhas desta planta.

\section{MATERIAL E MÉTODOS}

Classificação botânica. Amostras de Dodonea viscosa usadas no experimento, coletadas nos municípios de Osório e Porto Alegre, foram encaminhadas para a Fundação Zoobotânica do Rio Grande do Sul para análise morfológica.

Coleta da planta para estudo experimental. Foram utilizadas no experimento folhas de $D$. viscosa em brotação, coletadas no município de Osório no mês de junho de 2001, e folhas em períodos de inflorescência, coletadas no Campus do Vale (UFRGS), Porto Alegre, RS, durante os meses de setembro e outubro de 2002. As folhas verdes frescas foram separadas dos galhos e imediatamente administradas em doseúnica, por via oral, aos quatro bovinos. Amostras de folhas coletadas durante a inflorescência foram mantidas a sombra em temperatura ambiente, para desidratar, por um período de 15 dias e administradas por via oral, em dose única, ao quinto bovino.

\section{Quadro 1. Experimentos com folhas de Dodonea viscosa em bovinos}

\begin{tabular}{|c|c|c|c|c|c|c|}
\hline 3ovino & Raça & $\begin{array}{l}\text { Idade (meses) } \\
\text { /sexo }\end{array}$ & $\begin{array}{l}\text { Peso } \\
(\mathrm{kg})\end{array}$ & $\begin{array}{l}\text { Dose } \\
(\mathrm{g} / \mathrm{kg})\end{array}$ & $\begin{array}{l}\text { Início sinais } \\
\text { clínicos (h) }\end{array}$ & $\begin{array}{l}\text { Evolução } \\
\text { clínica (h) }\end{array}$ \\
\hline 1 & & 24/fêmea & 240 & 30 & & 31 \\
\hline 2 & & & & & & $7 r$ \\
\hline 3 & & & & 1 & Não adoeceu & \\
\hline 4 & & & 48 & 25 & $36 \mathrm{~h}$ & 1 \\
\hline 5 & Hereford & $30 /$ macho & 236 & $30^{a}$ & 13h30min & 4h30min \\
\hline
\end{tabular}

a Dose relativa à planta verde fresca.

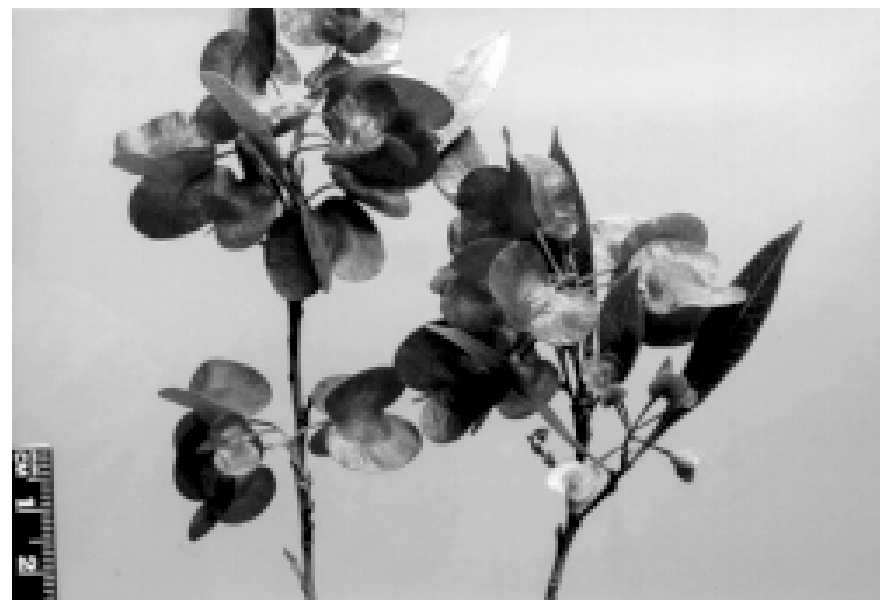

Fig. 2. Frutos de Dodonea viscosa, em setembro, Porto Alegre, RS.

Animais experimentais. Utilizaram-se cinco bovinos clinicamente sadios, sendo dois machos e três fêmeas, com idade entre 24 e 50 meses (Quadro 1). Durante 0 experimento os animais foram mantidos na Faculdade de Veterinária, UFRGS, em baias individuais, com água a vontade e feno de alfafa.

Estudo clínico e patológico. Exames clínicos foram realizados durante todo o experimento, com intervalos regulares. Avaliaram-se apetite, sede, temperatura retal, alterações cardio-respiratórias e modificações de comportamento, postura e movimento.

No Bovino 4, foram feitas coletas de sangue 12, 24 e 48 horas após a ingestão da planta, para verificação dos níveis da aspartato amino transferase (AST), g-glutamil transferase (GGT), fosfatase alcalina (SAP) e de glicose sérica.

Os animais foram necropsiados imediatamente após a morte e fragmentos de todos os órgãos foram coletados e fixados em formalina tamponada a 10\% Foram processadas rotineiramente para estudo histopatológico e corados pela técnica de hematoxilina e eosina (HE) (Prophet et al. 1992).

\section{RESULTADOS}

Dose tóxica e evolução clínica. Neste experimento com folhas de Dodonea viscosa foram observados sinais clínicos com morte nos Bovinos 1, 2, 4 e 5 que receberam as respectivas doses de $30 \mathrm{~g} / \mathrm{kg}$ de brotação, $30 \mathrm{~g} / \mathrm{kg}$ e $25 \mathrm{~g} / \mathrm{kg}$ de planta verde na fase de inflorescência e $30 \mathrm{~g} / \mathrm{kg}$ de planta seca. 0 Bovino 3 que recebeu $15 \mathrm{~g} / \mathrm{kg}$, não apresentou alterações clínicas.

A evolução do quadro clínico variou de 3 a 19 horas. 0 maior tempo entre a administração da planta e o início dos sinais clínicos foi de 45 horas (Bov. 1). 0 menor período entre a administração da planta e morte foi de 16 horas, no Bovino 5 que recebeu as folhas secas.

Sinais clínicos. 0 quadro clínico foi similar em todos os bovinos. Os principais sinais foram anorexia, apatia, redução dos movimentos ruminais, sudorese moderada, principalmente de cabeça e pescoço, leve tenesmo e dores abdominais. Notaram-se também tremores musculares, principalmente nos membros, que se evidenciavam após movimentação, dificul- 
Quadro 2. Dosagens séricas de aspartato amino transferase (AST), g-glutamil transferase (GGT), fosfatase alcalina (SAP) e de glicose, realizadas no Bovino 4 experimentalmente intoxicado por Dodonea viscosa

\begin{tabular}{ccccc}
\hline Amostra $^{a}$ & AST (U/L) & GGT(U/L) & SAP(U/L) & Glicose(mg/dl) \\
\hline Valor referênciab $^{b}$ & $78-132$ & $6.1-17,4$ & $<488$ & $45-75$ \\
12 horas & 345,5 & 41,8 & 77,1 & 62,7 \\
24 horas & 436,3 & 30,5 & 94,0 & 52,5 \\
48 horas & 577,0 & 35,2 & 178,1 & 9,2
\end{tabular}

aAs amostras têm como referência o horário de término da ingestão da planta. b(Cornelius 1989).

dade em permanecer em estação apoiando a cabeça na parede ou no cocho, relutância em caminhar, decúbito esternal, decúbito lateral com movimentos de pedalagem, coma e morte. 0 Bovino 2 apresentou agressividade 6 horas antes da morte.

Patologia clínica. Os níveis séricos das enzimas aspartato aminotransferase (AST), fosfatase alcalina (SAP) e g-glutamil transferase (GGT) e da glicose sérica foram analisados no Bovino 4 (Quadro 2). Alterações significativas ocorreram com os níveis da AST, da GGT e da glicose 48 horas após a administração da planta.

Achados macroscópicos. A lesão principal encontrava-se no fígado e consistia de acentuação do padrão lobular na superfície capsular e de corte, com áreas vermelho-escuras intercaladas com áreas vermelho-claras (Fig. 3). Notaram-se intensa repleção da vesícula biliar e leve edema de parede da vesícula, colédoco e duodeno. No rúmen havia grande quantidade de folhas de $D$. viscosa parcialmente digerida. 0 abomaso estava com mucosa intensamente avermelhada. Petéquias foram vistas no endocárdio e pericárdio, próximas ao sulco coronário e na serosa do omaso e intestino. 0 Bovino 4 apresentou icterícia moderada e conteúdo hemorrágico no cólon e ceco.

Achados microscópicos. Microscopicamente a principal lesão, em todos os animais necropsiados, afetava o fígado e consistia em necrose hepatocelular coagulativa, acompapanhada de congestão e hemorragia (Fig. 4). A necrose era centrolobular nos Bovinos 1 e 2 e massiva nos Bovinos 4 e 5. Na região centrolobular os hepatócitos estavam dissociados, encarquilhados, com maior eosinofilia citoplasmática, com os núcleos em picnose ou cariorrexia. Por vezes, havia desaparecimento de hepatócitos próximos à veia centrolobular. Nos casos com necrose massiva, a lesão se estendia aos hepatócitos periacinares restando uma a duas fileiras de hepatócitos morfologicamente não afetados. Alterações degenerativas, como vacuolização de hepatócitos e glóbulos eosinofílicos, foram observadas principalmente próximo ao limite da área com necrose e hepatócitos morfologicamente normais. (Quadro 3)

\section{DISCUSSÃO}

Neste estudo experimental foi obtido, pela administração de doses únicas de Dodonea viscosa a bovinos, o quadro clínico de insuficiência hepática aguda associada com necrose hepatocelular. As folhas foram tóxicas na fase de brotação,

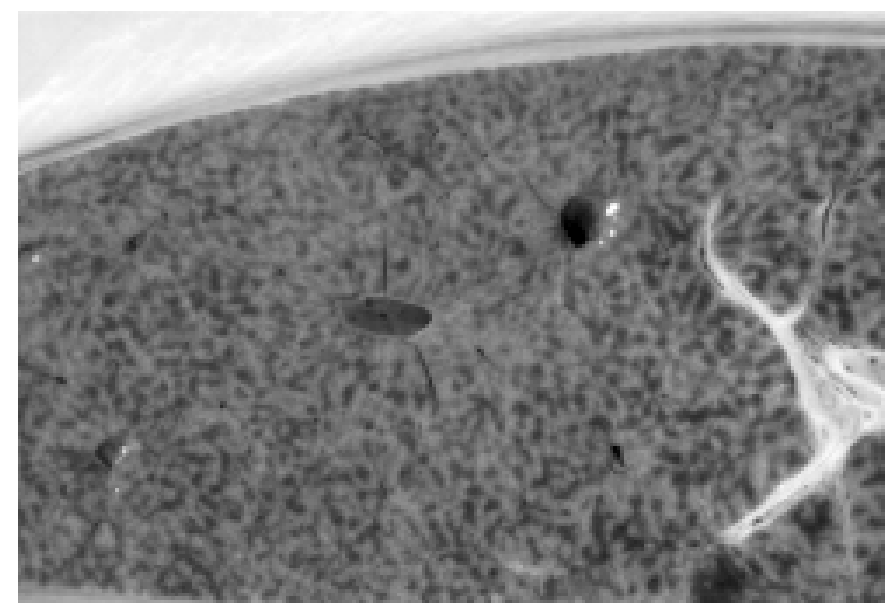

Fig. 3. Fígado com acentuação do padrão lobular, de aspecto de nozmoscada, na intoxicação experimental por Dodonea viscosa em bovinos. (Bov. 5)

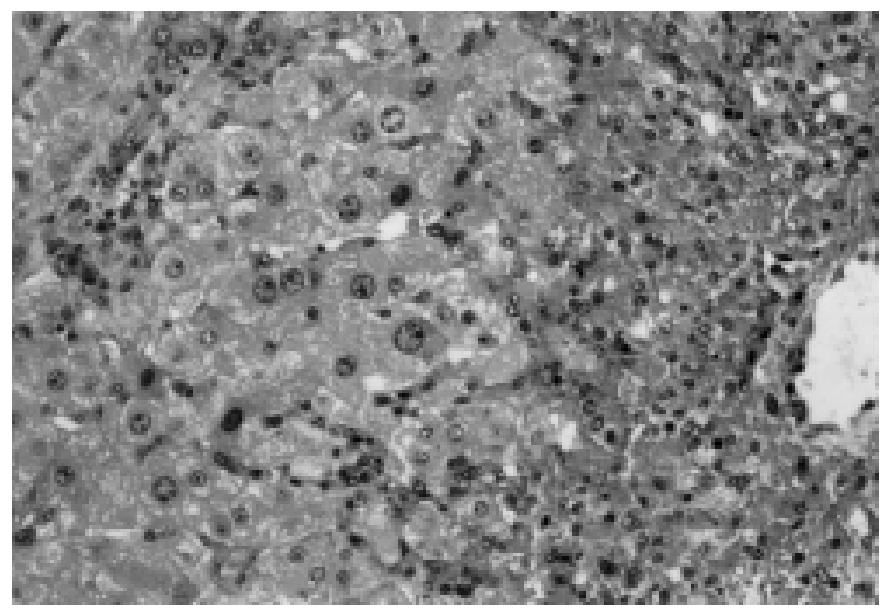

Fig. 4. Necrose de coagulação hepatocelular centrolobular com congestão e hemorragia, em bovino experimentalmente intoxicado por Dodonea viscosa. HE, obj. 40.

\section{Quadro 3. Classificação do grau das alterações microscópicas encontradas nos bovinos experimentalmente intoxicados por Dodonea viscosa}

\begin{tabular}{lcccc}
\hline \multicolumn{1}{c}{ Alterações } & Bov. 1 & Bov. 2 & Bov. 4 & Bov. 5 \\
\hline Necrose hepática & $+++\mathrm{a}$ & +++ & ++ & ++ \\
Esferóides eosinofílicos & + & + & +++ & ++ \\
Tumefação de hepatócitos & +++ & +++ & +++ & ++ \\
Hemorragias & ++ & ++ & +++ & +++
\end{tabular}

$\overline{a_{+}++ \text {Lesão acentuada }},++$ moderada, + leve, - ausente.

inflorescência e quando secadas a sombra. 0 quadro clínico e as lesões foram similares àquelas encontradas na intoxicação natural por D. viscosa em bovinos (Colodel et al. 2003). Dos cinco animais que ingeriram a planta, quatro morreram. A planta verde se mostrou tóxica a partir de $25 \mathrm{~g} / \mathrm{kg}$. 0 início dos sinais clínicos após a ingestão de $D$. viscosa variou de 13ho- 
ras e 30 minutos a 45 horas e a evolução clínica foi de 3 a 19 horas.

Os principais achados clínicos de insuficiência hepática aguda são comuns para as plantas que causam necrose hepatocelular e foram descritos em casos de bovinos intoxicados com outras plantas tóxicas, como Sessea brasiliensis (Canella et al. 1968), Cestrum laevigatum (Döbereiner et al. 1969), Cestrum parqui (Riet-Correa et al. 1986), Vernonia mollissima (Döbereiner et al. 1976, Gava et al. 1987), Vernonia rubricaulis (Tokarnia \& Döbereiner 1982), Cestrum corymbosum var. hirsutum (Gava et al. 1991), Cestrum intermedium (Gava et al. 1996) e Xanthium cavanillesii (Driemeier et al. 1999).

O princípio tóxico de $D$. viscosa associado com a necrose hepatocelular, não é conhecido. As alterações patológicas, encontradas no fígado dos bovinos desse experimento são as que caracterizam a lesão hepática aguda de origem tóxica, foram descritas na intoxicação natural por D. viscosa (Colodel et al. 2003) e ocorrem associadas a outras hepatotoxicoses agudas em bovinos (Martin et al. 1986, Witte et al. 1990, Méndez et al. 1994, Driemeier et al. 1999, Colodel et al. 2000, Tokarnia et al. 2000).

Agradecimentos.- À Drạ Olinda Leites Bueno, da Fundação Zoobotânica do Rio Grande do Sul, pela classificação botânica de Dodonea viscosa. Às Sras. Marilia Terezinha de Oliveira Belmonte e Ângela Rosaura Belmonte de Souza, do Laboratório de Patologia Veterinária, UFRGS, pelos processamentos e pelas colorações histológicas.

\section{REFERÊNCIAS}

Canella C.F.C., Tokarnia C.H. \& Döbereiner J. 1968. Intoxicação por Sessea brasiliensis Toledo em bovinos. Pesq. Agropec. Bras. 3:333-340.

Colodel E. M., Driemeier D. \& Pilati C. 2000. Intoxicação experimental pelos frutos de Xanthium cavanillesii (Asteraceae) em bovinos. Pesq. Vet. Bras. 20(1):31-38.

Colodel E.M., Traverso S.D., Seitz A.L., Oliveira F.N., Driemeier D. \& Gava A. 2003. Spontaneous poisoning by Dodonea viscosa (Sapindaceae) in cattle. Vet. Human Toxicol. 45(3):147-148.

Cornelius C.E. 1989. Liver Function, p. 364-397. In: Kaneko J.J. (ed.) Clinical Biochemistry of Domestic Animals. $4^{\text {th }}$ ed. Academic Press, San Diego

Döbereiner J., Tokarnia C. H. \& Canella C. F. C. 1969. Intoxicação por Cestrum laevigatum Schlecht., a causa de mortandades em bovinos no Estado do Rio de Janeiro. Pesq. Agropec. Bras. 4:165-193.

Döbereiner J., Tokarnia C. H. \& Purisco E. 1976. Vernonia mollissima, planta tóxica responsável por mortandades de bovinos no sul do Mato Grosso. Pesq. Agropec. Bras. 11:49-58.

Driemeier D., Irigoyen L. F., Loretti A. P. Colodel, E.M. \& Barros C. S. L. 1999. Intoxicação espontânea pelos frutos de Xanthium cavanillesii (Asteraceae) em bovinos no Rio Grande do Sul. Pesq. Vet. Bras. 19(1):12-18.

Gava A., Peixoto P. V. \& Tokarnia C. H. 1987. Intoxicação experimental por Vernonia mollissima em ovinos e bovinos. Pesq. Vet. Bras. 7(2): 33-41.

Gava A., Stolf L., Pilati C., Neves D. S. \& Viganó L. 1991. Intoxicação por Cestrum corymbosum var. hirsutum (Solanaceae) em bovinos no estado de Santa Catarina. Pesq. Vet. Bras. 11(4):71-74.

Gava A., Stolf L., Varaschin M. S., Neves D. S., Tigre A. P. \& Lessmann F. 1996. Intoxicação por Cestrum intermedium (Solanaceae) em bovinos. Pesq. Vet. Bras. 16(4): 117-120.

Martin T., Stair E.L. \& Dawson L. 1986. Cocklebur poisoning in cattle. J. Am. Vet. Med. Assoc. 189(5):562-563.

Méndez M. C., Santos R. C. \& Riet-Correa F. 1998. Intoxication by Xanthium cavanillesii in cattle and sheep in southern Brazil. Vet. Human Toxicol. 40(3):144-147.

Mors B.W., Rizzini C.T. \& Pereira N.A. 2000. Medicinal Plants of Brazil. Reference Publications, Algonac. 501p.

Pesman M.W. \& Ervin E.A. 2002. Meet the natives: A Beginner's field Guide to Rocky Mountain Wildflower, Trees, and Scrubs. Roberts Rinehart Publishers, Denver. 248p.

Prophet E.B., Mills B., Arrington J.B. \& Sobin L.H. 1992. Laboratory Methods in Histotechnology. Armed Forces Institute of Pathology, Washington, DC. 279p.

Riet-Correa F., Schild A.L. \& Méndez M.C. 1986. Intoxicação por Cestrum parqui (Solanaceae) em bovinos no Rio Grande do Sul. Pesq. Vet. Bras. 6(4): 111115.

Reitz R. 1980. Flora Ilustrada Catarinense: Sapindáceas. Herbário Barbosa Rodrigues, Itajaí, SC. 156p.

Simões C. M. O., Mentz L. A., Schenkel E. P., Irgang B. E. \& Stehmann J. R. 1989. Plantas da Medicina Popular no Rio Grande do Sul. $3^{\text {a }}$ ed. Editora da UFRGS, Porto Alegre.174p.

Tokarnia C.H. \& Döbereiner J. 1982. Intoxicação de bovinos por Vernonia rubricaulis (Compositae) em Mato Grosso. Pesq. Vet. Bras. 2(4):143-147.

Tokarnia C.H., Döbereiner J. \& Peixoto P.V. 2000. Plantas Tóxicas do Brasil. Editora Helianthus, Rio de Janeiro. 320p.

Witte S.T., Osweiler G.D., Stahr H.M. \& Mobley G. 1990. Cocklebur toxicosis in cattle associated with the comsumption of Xanthium strumarium. J. Vet. Diagn. Invest. 2(4): 263-267. 